\title{
puentes...
}

Piedra sobre piedra, en pesados arcos de poca luz, salvan valles $y$ ríos los puentes romanos que publicamos en un nuevo capítulo de la «Historia del puente en España».

Ante ellos, y a modo de prólogo, publicamos otros puentes de hoy, construídos en menos tiempo, con menos peso y con mayores luces.

El puente de Mackinac, reciente obra de Steinman, salva $1.158 \mathrm{~m}$ de luz en su tramo central colgado, mientras que la longitud total del puente es de unos cinco kilómetros.

El puente Volta se resuelve con tablero colgado, mediante péndolas verticales, de un gran arco, en triagulación metálica, que salva $256 \mathrm{~m}$ entre sus dos articulaciones de apoyo.

El puente de Saint Maurice es una audaz solución de hormigón pretensado, que salva $116 \mathrm{~m}$ de luz. El 'puente de Troy, más que por su luz, de sólo $45 \mathrm{~m}$,

ofrece interés por el sistema adoptado de pretensar sus vigas en celosía metálica, ya construídas, para reforzar su resistencia.

El progreso de la ciencia y de la técnica hace posible la creación de obras cada vez más sorprendentes, a las que es de desear una duración, al menos, como la de esos modestos arcos pétreos. 


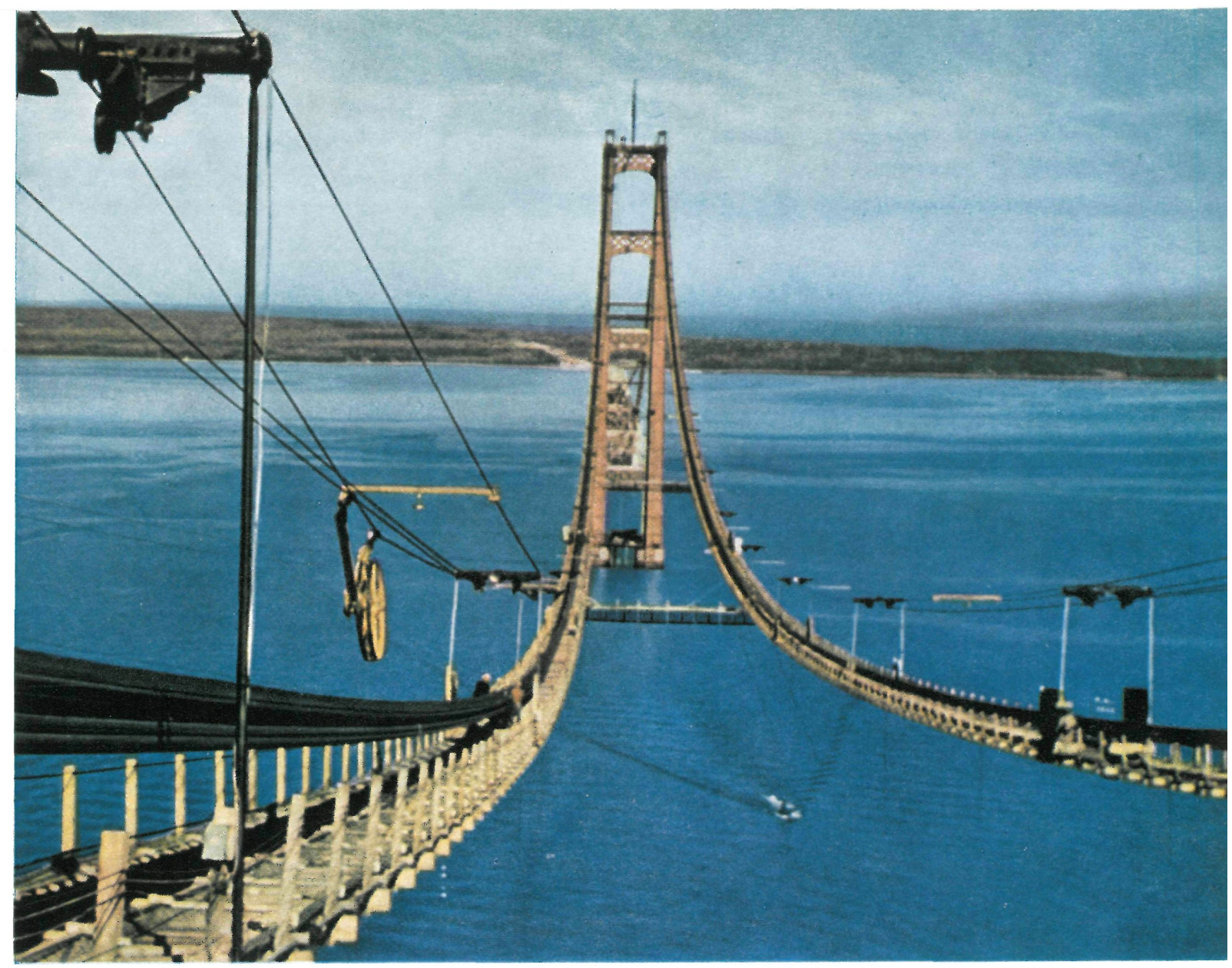

$564 \cdot 11$

\section{puente de Mackinac}

DAVID B. STEINMAN

En el número 82 de nuestra revista se publicó un trabajo que se refería únicamente a la infraestructura de esta importante obra. En esta segunda parte damos a conocer las características más sobresalientes de la propia superestructura, estabilidad,

métodos constructivos empleados en el montaje y el procedimiento que se ha seguido

para lograr colocar los cables, de 12.500 toneladas de peso total,

sobre las dos torres del tramo central, de $1.158 \mathrm{~m}$ de luz, método que ha consistido en formar los cables,

partiendo de las bobinas de alambre, en la propia obra.

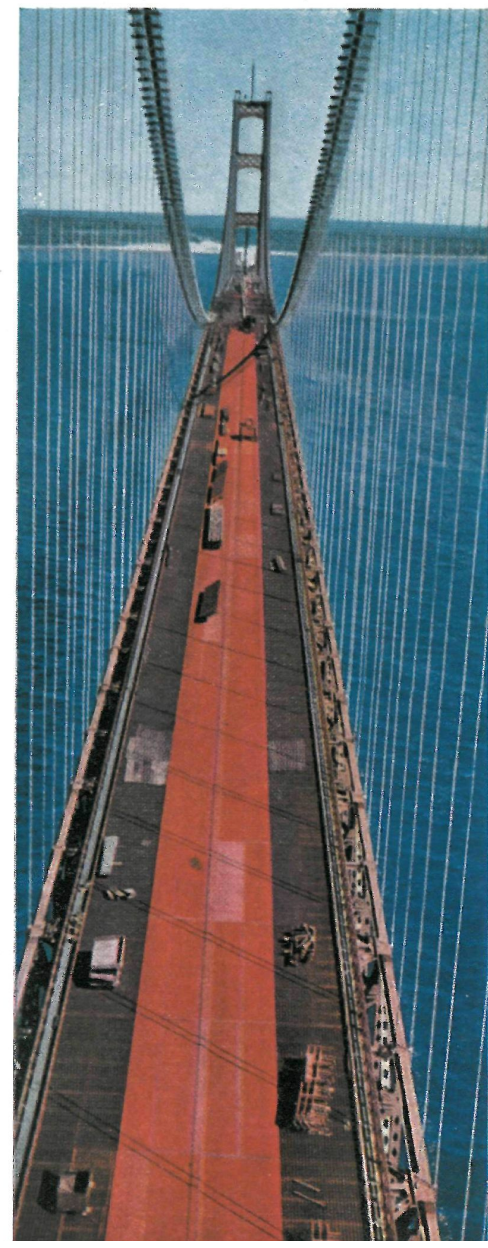




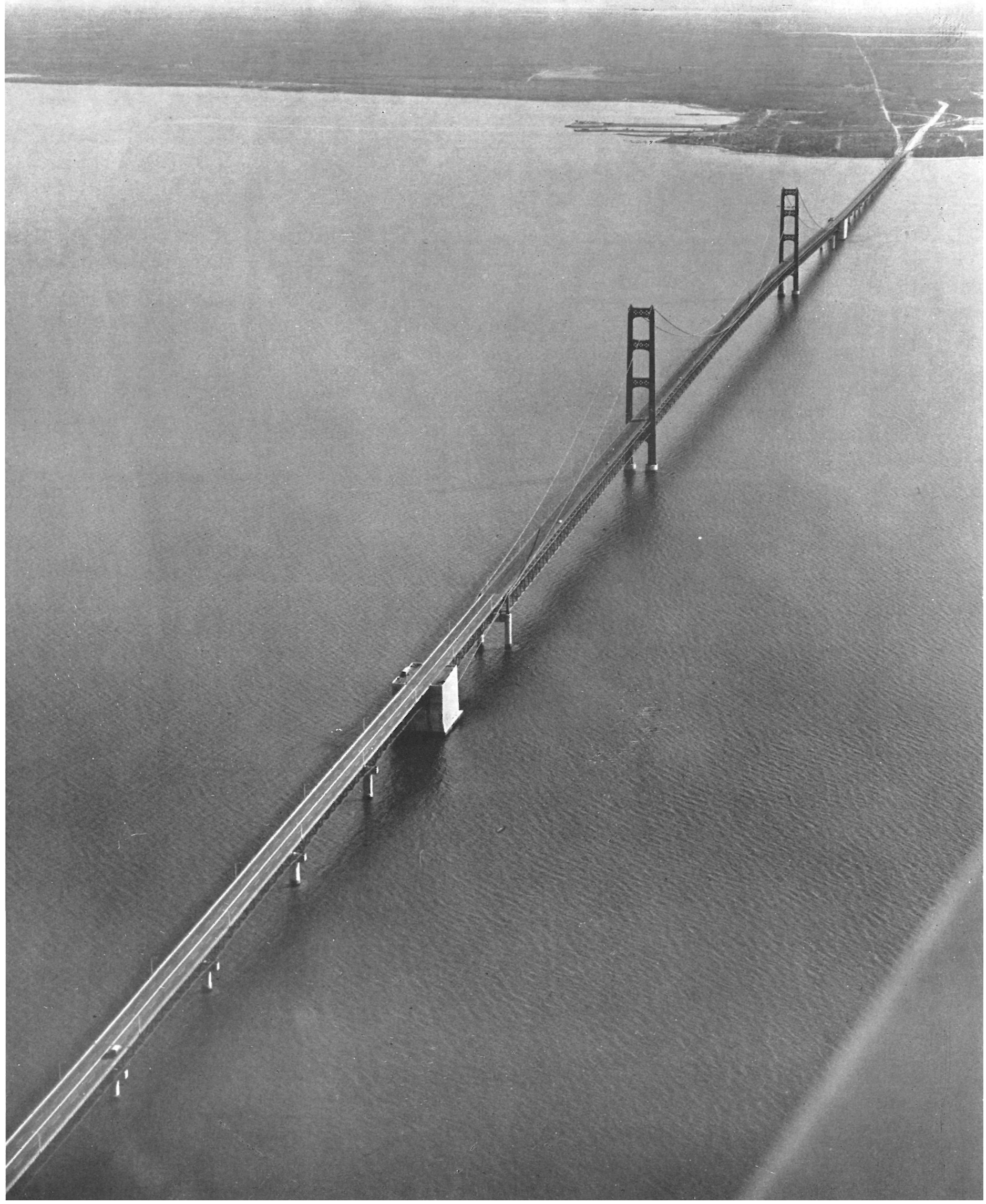

(C) Consejo Superior de Investigaciones Científicas Licencia Creative Commons 3.0 España (CC-by) 


\section{Situación general y objeto del puente}

El puente de Mackinac salva el estrecho del mismo nombre en la parte septentrional del Estado de Michigán (EE. UU.) donde la unión entre los lagos de Michigán y Hurón forman un relativamente angosto paso entre dos penínsulas: la de Mackinac al sur, y, enfrente, la de San Ignacio.

Antes de construir el puente, actualmente ya en servicio, el paso de una a otra parte del estrecho se efectuaba por medio de transbordadores que, aún siendo de gran capacidad, resultaban ser insuficientes para hacer frente a un voluminoso tráfico rodado, motivando grandes esperas y molestias consitráfico rodado, motivando grandes esperas y molestias consiguientes a los numerosos turistas y pescadores que los utilizaban casi continuamente. Esta fué la causa original que dió de paso superior, cuyo presupuesto fué de cien millones de dólares.

La distancia minima que separa las dos penínsulas en que estriba el puente es de unos ocho kilómetros; y la profundidad de aguas en el canal navegable, de consideración. La gran profundidad creaba grandes dificultades para lograr un gran profundidad creaba grandes dificultades para lograr un de las grandes luces en el tramo central, pues de operar así se aliviaba notablemente la construcción de cimientos y soportes.

\section{Estabilidad de la estrucfura}

Aunque la longitud total del puente sea de unos cinco kilómetros, el interés general de esta importante estructura se centra en su tramo central, de $1.158 \mathrm{~m}$ de luz, ya que los accesos están constituídos por un gran número de tramos de accesos están constituidos por un gran numero de tramos de que van de los bloques de anclaje de cables a las torres, no tienen más que $144 \mathrm{~m}$ de luz.

Dada la gran luz del tramo central, la solución general aceptada desde el primer momento fué la de suspender el tablero partiendo de dos potentes cables de los que partirían las péndolas de fijación del tablero en su debida posición.

El fenómeno ocurrido en el puente Tacoma, cuya descripción y estudio de causas ha sido descrito en un trabajo publicado en la Monografía núm. 83 de la colección del Instituto Técnico de la Construcción, advirtió al ingeniero encargado de la redacción de este proyecto, autor de este trabajo, los graves peligros que se derivan de un estudio incompleto de la estabilidad aerodinámica de una obra suspendida.

Entre un abuso excesivo de materiales para hacer frente a influencias no previstas o ignoradas y la concepción de una estructura capaz de resistir a un efecto determinado, existe una solución racional en la que se tiende a suprimir las disposiciones de elementos que pudieran ser fuente de efectos posiciones de elementos que pudieran ser fuente de efectos nocivos y peligrosos, en forma que, sin perder su trabajo funcional estructuralmente hablando, no permitan se puedan desarrollar dichos efectos, es decir, estudiar y suprimir las causas orientación ha servido al proyectista como base para encontrar una justa solución al delicado problema de la estabilidad aerodinámica en los puentes colgantes de gran luz.

El fenómeno de la inestabilidad aerodinámica no constituye un misterio pudiendo estudiarse dentro de un análisis racional y científico en toda su pureza. No todas las secciones transversales se comportan de una forma similar en el puente, por versales se comportan de una forma similar en el puente, por lo que hay que diferenciarlas respecto a su comportamiento tical, torsional y combinado. Las características aerodinámicas tical, torsional y combinado. Las características aerodinámicas de una estructura se pueden estudiar en un sencillo modelo
reducido sin necesidad de llevar al túnel soplante grandes modelos y seguir el método de aproximaciones y correcciones sucesivas de acuerdo con los resultados que se van obteniendo en los ensayos; operaciones, todas ellas, que requieren un gran período de tiempo.

El comportamiento aerodinámico de un puente de esta naturaleza se puede determinar analizando los gráficos del empuje ascensional y par de torsión de las diferentes secciones ensayadas en un túnel soplante pequeño. El gradiente de estas curvas es el dato que caracteriza el grado de estabilidad.

La sección ideal, desde el punto de vista aerodinámico, es la que corresponde a curvas de pendiente cero o nula. Esta pendiente en los gráficos de esfuerzos ascensionales y de torsión indica que estas fuerzas no existen $\mathrm{y}$, por tanto, que no debe esperarse aparezcan las oscilaciones aerodinámicas.

La estabilidad aerodinámica de un puente se puede asegurar con "simples" modificaciones introducidas en las secciones rar con "simples" modificaciones introducidas en las secciones ordinarias de puentes. Las modificaciones que han de introducirse en las secciones ordinarias de puentes han sido causa
de una serie de trabajos publicados a partir de 1940, año en que ocurrió el fenómeno de resonancia en el puente Tacoma.

Las medidas tomadas para lograr una gran estabilidad aerodinámica consisten, esencialmente, en dejar grandes espacios libres o huecos entre las dos grandes celosías laterales de rigidez. La zona más afectada en la provocación de esfuerzos diferenciales ascensionales, y, por tanto, de torsión, tanto mayores cuanto mayor es la excentricidad, corresponde a la parte excuanto mayor es la excentricidad, corresponde a la parte externa del tablero, por lo que en esta región se han dejado grandes huecos. De las cuatro bandas de circulación de la calzada, dos para cada dirección de la circulación, separadas por un andén central, las dos bandas exteriores corresponden a una pavimentación asfáltica continua, mientras que, en las tuído por una especie de rejilla que apenas si opone resistencia al paso del viento. Esta disposición permite un equilibrio de presiones entre las que afectan la parte superior $\mathrm{e}$ inferior del tablero, así como suprimir radicalmente el esfuerzo diferencial, la excentricidad y torsión, logrando, simple y sencillamente, lo que se ha dado en llamar estabilidad aerodinámica. Para que exista un esfuerzo de este tipo y su efecto, es necesario una superficie sobre la que la presión y su efecto, es necesario una superficie sobre la que la presión
ejerza su influencia. Este es escuetamente el origen de la disposición adoptada.

Los gráficos de esfuerzos ascensionales se obtienen manteniendo un modelo en el túnel soplante $\mathbf{y}$ anotando los esfuerzos ascensionales que corresponden a los diferentes ángulos de ataque del viento en el túnel. Estos ángulos se logran inclinando el modelo mantenido estáticamente en el túnel durante los ensayos. La pendiente de la curva obtenida en el diagrama de referencia representa el grado de aumento del esfuerzo ascensional según el ángulo de ataque. Al dibujar esfuerzo ascensional según el ángulo de ataque. Al dibujar estos gráficos debe tenerse en cuenta que es el modelo el
inclinado, y no el viento $y$, por tanto, se ha de introducir la correspondiente corrección por falta de conformidad.

La pendiente de la curva del gráfico tiene un sentido característicamente significante. Una pendiente negativa caso de un aumento de ángulo de ataque y disminución de esde un aumento de angulo de ataque y disminución de esfuerzo ascensional, representa un estado peligroso, "inestabilidad catastrófica" en las oscilaciones de sentido vertical. Una
estructura sometida a esta alternativa, nos lleva a una osci-
lación acumulativa conducente a amplitudes ruinosas en el plano vertical. Este tipo de curva sólo ha aparecido en un puente moderno, pero se tomaron las correspondientes medidas de seguridad para restablecer la estabilidad aerodinámica apropiada.

Una pendiente positiva en el gráflco de empujes ascensionales identifica las secciones "estables", asi llamadas para distinguirlas de las denominadas "catastróficamente inestables". Esta distinción es clara en el caso de secciones geométricas de forma lenticular, en las que la profundidad excede a la altura. Sin embargo, en las secciones transversales de los puentes, una sección "estable", es decir, una de pendiente positiva en el gráfico de empujes ascensionales, se halla influenciada por oscilaciones aerodinámicas limitadas dentro de cierto campo crítico de la velocidad del viento Estas oscilaciones aerodinámicas aparecen, corrientemente, con bajas velocidades del viento. Aunque no son "catastróficas", estas oscilaciones que dan lugar las bajas velocidades 
son alarmantes por acortar la vida de la estructura debido a la fatiga de materiales. A este tipo de oscilaciones, comúnmente conocidas con el nombre de "galopantes", pertenecen las que se desarrollaron en el, desde sus primeros momentos mal predestinado, puente de Tacoma (1940), durante los primeros cuatro meses de su vida.

En los dos casos-la inestabilidad catastrófica correspondiente a una pendiente negativa en el gráfico de empujes ascensionales y la estabilidad limitada que caracteriza a una pendiente positiva en el referido gráfico- el grado de inestabilidad es proporcional a la pendiente de la curva del gráfico de empujes; y como esta relación es aplicable a todos gráfico de empujes; y como esta relación es aplicable a todos los gráficos, aun con pendientes positivas o negativas, la
conclusión se presenta claramente: la sección ideal de un conclusión se presenta claramente: la sección ideal de un
puente es aquella cuya pendiente es nula en el gráfico correspondiente de empujes ascensionales.

La pendiente obtenida en la curva del gráfico correspondiente a los empujes en el puente Mackinac es $+0,03$, es decir, virtualmente nula. Este grado de perfección no ha sido alcanzado ni aproximadamente hasta la actualidad en otras estructuras de este género. Comparando grados de estabilidad, la del puente Mackinac es unas 350 veces más que la del Golden Gate tanto en lo que respecta a la oscilación vertical como a la llamada galopante.

En la hipótesis de que la nieve o hielo llegara a cerrar la parte hueca del reticulado de la zona central de la calzada, la estabilidad relativa respecto al Golden Gate sería unas $51 / 2$ veces mayor, $y$, además, el estado que crearía dicha obstrucción por nieve o hielo mejoraría las condiciones de amortiguamiento de oscilaciones, acción esta última que contrarrestaria, por lo menos parcialmente, el aumento de pendiente en el gráfico debido al taponamiento de la rejilla del tablerón en la zona central.

Los ensayos realizados últimamente en el túnel soplante con grandes modelos han confirmado la gran estabilidad del puente Mackinac desde el punto de vista aerodinámico, tanto dentro de un estado oscilante vertical con velocidades variables del viento como bajo todos los ángulos de ataque de éste.

Similarmente al fenómeno de la oscilación en el plano vertical, el par de torsión se caracteriza también por la pendiente de la curva correspondiente a estos momentos. Si es ideal, pues se hallan eliminadas todas las fuerzas y momentos que pudieron inducir a una ampliación de oscilaciones que aumentarían progresivamente el par de torsión, o, como se diría más gráficamente, "retorcer" la estructura.

El incremento logarítmico o coeficiente de ampliación de la inestabilidad debida a la torsión resulta ser proporcional a la pendiente de los gráficos del par estático de torsión. El análisis matemático completo utiliza, además, un gráfico suplementario del par estático de torsión, obtenido partiendo de un modelo curvo de la sección transversal sometida a ensayo.

Además del valor de la magnitud de la pendiente, su dirección tiene un rignificado crítico. Si esta dirección es negativa, es decir, hacia abajo, el gráfico correspondiente a un estado de es decir, hacia abajo, el gráfico correspondiente a un estado de inestabilidad torsional catastrófica. Este es el tipo peligroso que fueron la causa de la destrucción del puente de Tacoma el 7 de noviembre de 1940 .

Si la pendiente es positiva en el gráfico correspondiente, nos hallamos ante un caso de estabilidad de la sección sometida a torsión, esto no obstante, sometida a un cierto grado de oscilaciones que no se pueden calificar de catastróficas, y su amplitud se halla limitada de unos centímetros hasta un metro, aproximadamente, presentándose con velocidades bajas del viento. Aun tratándose de oscilaciones limitadas son molestas y alarmantes, porque debilitan la estructura son molestas y alarm
por efecto de fatiga.

De cuanto llevamos dicho referente a los efectos de torsión por causas de oscilaciones, se deduce que la sección ideal es aquella que tiene pendiente nula en el gráfico de momen- tos de torsión, criterio este que corre un paralelismo con las conclusiones a que se ha llegado al tratar de los gráficos de esfuerzos ascensionales.

Si tanto en el gráfico de empujes ascensionales como en el de momentos de torsión la dirección de la pendiente es positiva, la sección se hace vulnerable por el efecto combinado de las oscilaciones que pudieran sincronizarse vertical y torsionalmente. Así, pues, gráficos con pendiente nula en ambos casos o ligera pendiente positiva en el gráfico de esfuerzos ascensionales y una pequeña pendiente negativa en el gráfico de momentos de torsión constituyen soluciones ideales para las secciones de puentes suspendidos, ya que ideales para las secciones de puentes suspendidos, ya que, torsional y combinada.

\section{Canto o altura de las jácenas \\ - celosías de rigidez}

La ignorancia en que se pudiera incurrir al proyectar un puente en el que entra en juego la estabilidad aerodinámica, - los defectos observados a este respecto en una obra ya existente, pueden corregirse con la cruda y extravagante idea de aumentar exageradamente el canto o altura de las celosías o jácenas de rigidez.

En un manual de ingeniería se pretende poder proyectar un puente colgante con un canto de $1 / 40$ a $1 / 60$ de la luz un puente colgante con un canto de $1 / 40$ a $1 / 60$ de la luz lidad del desarrollo de oscilaciones "galopantes". El principio es técnicamente correcto, pero supone el sacrificio de una buena parte de materiales para cubrir los riesgos en que la ignorancia pudiera incurrir. Salvo en las pequeñas luces, el procedimiento daría torpes, pesadas y extravagantes estructuras.

Con esta finalidad se ha obtenido un sistema de cálculo en el que la fórmula final dé la altura apropiada de las jácenas de rigidez para varias luces, y se añade una cláusula de salvedad que dice: "a menos que la estabilidad pueda asegurarse de otra forma".

La utilización de esta fórmula en una luz de $1.158 \mathrm{~m}$, que es la del tramo central de este puente, correspondería un canto de $14 \mathrm{~m}$ para las celosías de rigidez, es decir, 1/82 en números redondos de la luz. Pero aún así, una altura de $14 \mathrm{~m}$ para las jácenas resultaría extravagante y una pérdida inútil para materiales. La altura finalmente adoptada perdida inutil dervadora, es de $11,50 \mathrm{~m}$, que corresponde a $1 / 100$ de la luz Teniendo en cuenta la gran estabilidad aerodinámica conseguida con la disposición apropiada de elementos estructurales y tablero, de haberse adoptado un canto menor, la estructura habría permanecido dentro de los límites de seguridad admisibles. El ingeniero Glenn B. Woodruff, asociado en la redacción del proyecto al autor, dijo que, debido a la seguridad aerodinámica lograda, él se hubiera conformado satisfactoriamente con sólo un canto de 3,70 metros.

La reducción racional de la altura de las vigas de rigidez en una luz de esta importancia como la del tramo central del puente Mackinac tiene gran importancia económicamendel puente Mackinac tiene gran importancia económicamenestabilidad aerodinámica, las eccnomías podían haberse eleestabilidad aerodinámica, las eccnomías podían haberse ele-
vado a unos millones de dólares, pero por un lado el criticismo $y$ por otro la falta de interpretación del fenómeno de estabilidad aerodinámica, y el tener que conformar al propietario del puente, público en general y compañías de seguros, han exigido al autor del proyecto presentar una disposición estructural ultraconservadora para que fuera aceptada la realización de la obrz.

Con la introducción de dos planos de arriostramiento, uno a la altura de la cabeza superior de las celosías de rigidez $y$ el otro en la inferior, arriostramiento que se extiende en toda la longitud del tramo central, se ha conseguido una sección hueca rectangular que asegura una gran rigidez a la torsión de la estructura y, a su vez, un aumento de la capacidad de amortiguamiento en torsión. 

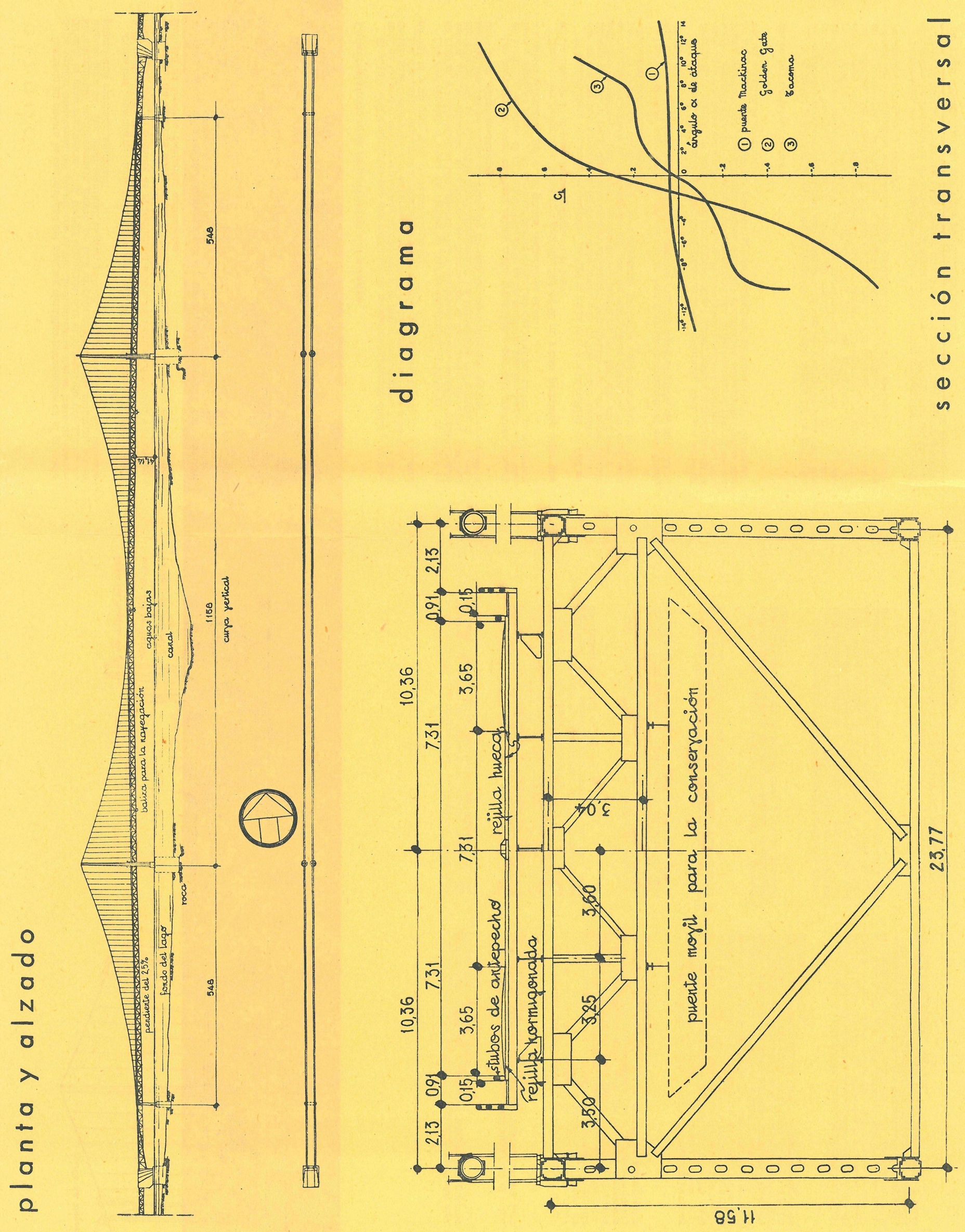

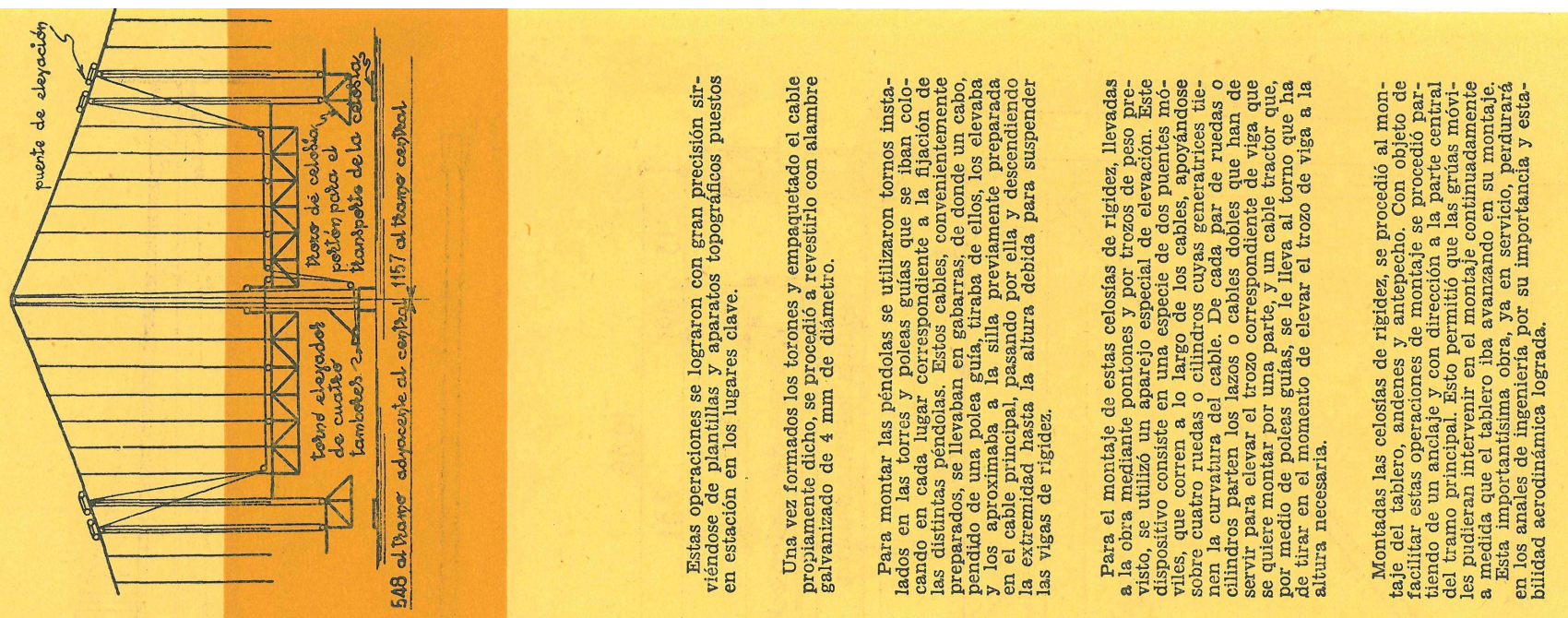

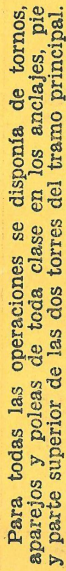

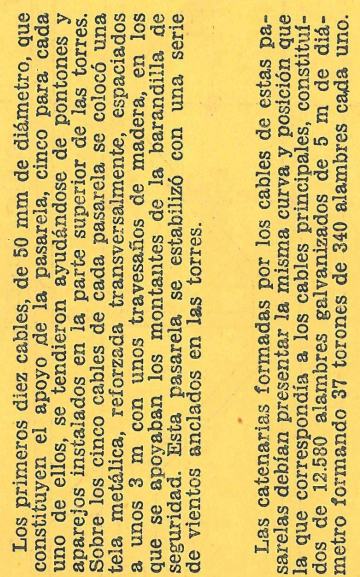

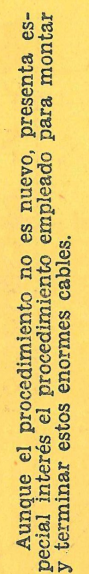

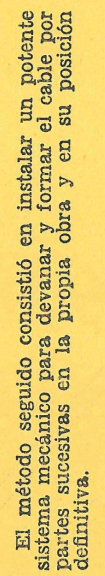

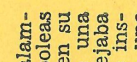
\%

\%

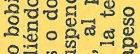

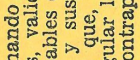

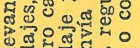

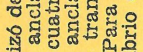

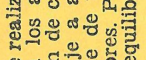

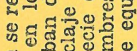

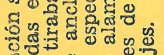

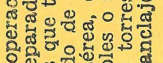

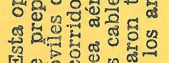

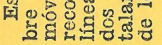

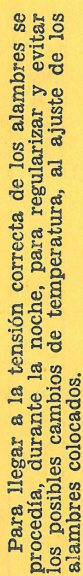

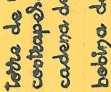

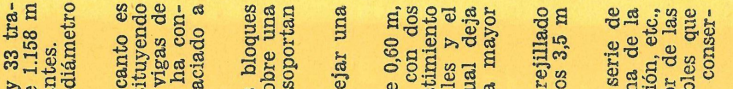

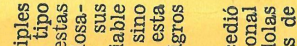

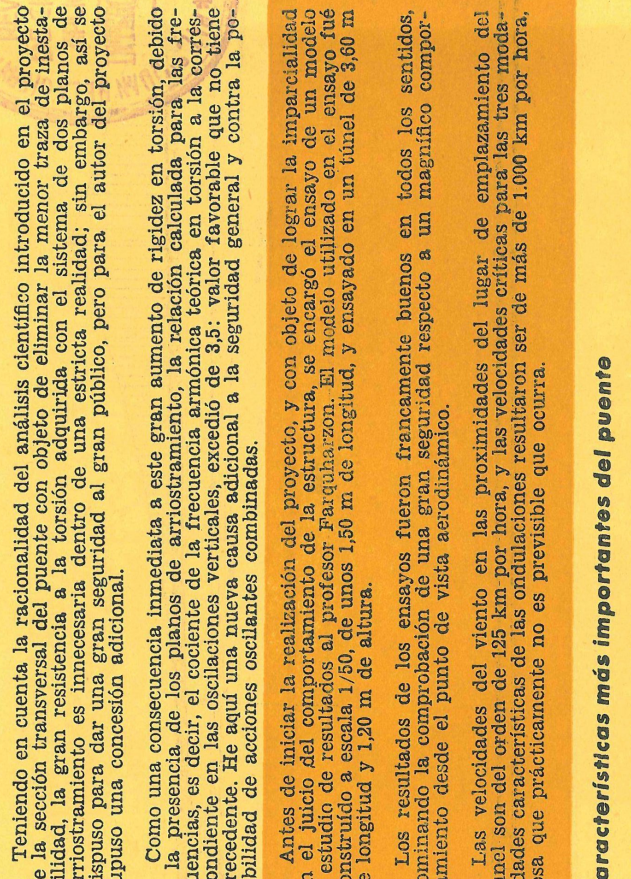

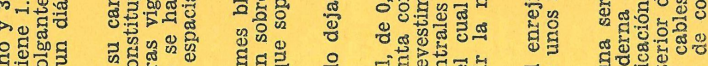

等:

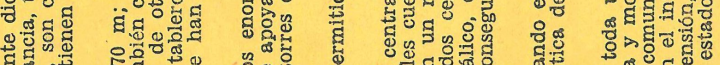

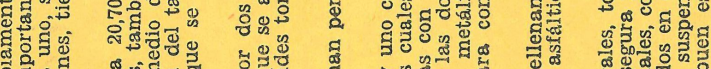

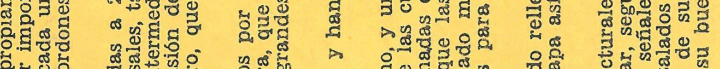

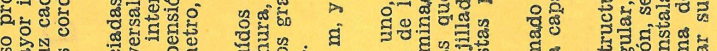

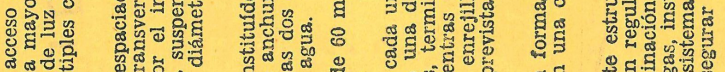

\&

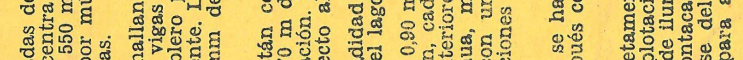

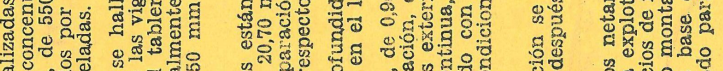

a.

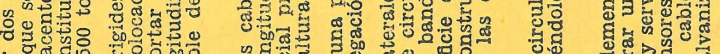
次

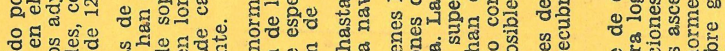

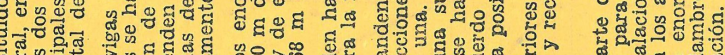

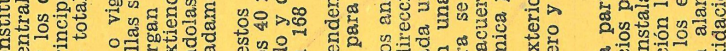

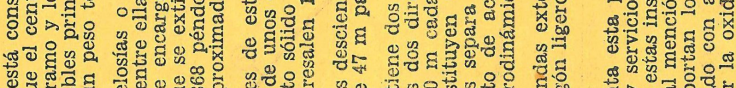

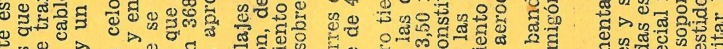

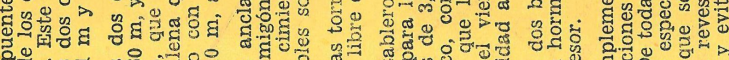

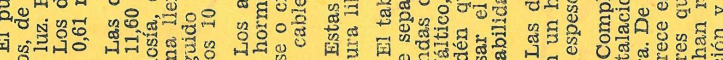

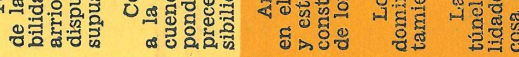

is 